\title{
A Neural Marker of Perceptual Consciousness in Infants
} Sid Kouider et al.

Science 340, 376 (2013);

DOI: $10.1126 /$ science.1232509

This copy is for your personal, non-commercial use only.

If you wish to distribute this article to others, you can order high-quality copies for your colleagues, clients, or customers by clicking here.

Permission to republish or repurpose articles or portions of articles can be obtained by following the guidelines here.

The following resources related to this article are available online at www.sciencemag.org (this information is current as of April 18, 2013 ):

Updated information and services, including high-resolution figures, can be found in the online version of this article at:

http://www.sciencemag.org/content/340/6130/376.full.html

Supporting Online Material can be found at:

http://www.sciencemag.org/content/suppl/2013/04/17/340.6130.376.DC1.html

This article cites 27 articles, 8 of which can be accessed free:

http://www.sciencemag.org/content/340/6130/376.full.html\#ref-list-1

This article appears in the following subject collections:

Neuroscience

http://www.sciencemag.org/cgi/collection/neuroscience 
intensity in the ALG-1 IP suggests that only a subpopulation of LIN-41 is in complexes with ALG-1. Similarly, IP of GFP-tagged LIN-41 pulled down a subset of the ALG-1 protein population (fig. S10B). IP of small subsets of protein populations with components of microRNA-induced silencing complex has precedents $(15,19)$.

Even though let-7 levels decline significantly by day 4 into the adult stage (fig. S11, A and B), AVM axon regeneration remained significantly enhanced in aged animals that either overexpress lin-41 or contain let-7 mutations (fig. S11, $\mathrm{C}$ and D), which indicates that the let-7/lin-41 pathway regulates axon regeneration even in aged AVM. Furthermore, let-7 mutations significantly enhance, whereas lin-41 mutations significantly reduce, axon regeneration in several neurons that coexpress both genes, which suggests that the role of let-7/lin-41 in regulating axon regeneration can be extended beyond AVM neurons (fig. S12).

In this study, we show that let-7 contributes to a developmental decline in AVM axon regeneration. We identify LIN-41 as an important AVM axon regeneration-promoting factor. let-7 represses lin-41 expression to inhibit AVM axon regeneration in older neurons. Our results suggest a negative regulatory loop between let-7 and lin-41 (Fig. 4G). In younger neurons, lin-41 inhibits let-7 expression through negative regulation of ALG-1. In older neurons, up-regulation of let-7 overcomes the lin-41 inhibition and, in turn, represses the lin- 41 expression through the lin-41 3' UTR. Like C. elegans, mammals also exhibit a developmental decline in axon regeneration. Our findings suggest that it may be possible to enhance axon regeneration after injury through therapeutic inhibition of the let-7 microRNA in neurons and, thereby, to restore their youthful regenerative capacity.

\section{References and Notes}

1. H. Chiu, A. Alqadah, C.-F. Chuang, C. Chang, Cell Adhes. Migr. 5, 387 (2011).

2. Z. Wu et al., Proc. Natl. Acad. Sci. U.S.A. 104, 15132 (2007).

3. C. V. Gabel, F. Antoine, C.-F. Chuang, A. D. T. Samuel, C. Chang, Development 135, 3623 (2008).

4. M. Hammarlund, P. Nix, L. Hauth, E. M. Jorgensen, M. Bastiani, Science 323, 802 (2009).

5. J. L. Goldberg, M. P. Klassen, Y. Hua, B. A. Barres, Science 296, 1860 (2002).

6. M. Boehm, F. Slack, Science 310, 1954 (2005).

7. R. C. Lee, R. L. Feinbaum, V. Ambros, Cell 75, 843 (1993).

8. B. Wightman, I. Ha, G. Ruvkun, Cell 75, 855 (1993).

9. A. E. Pasquinelli et al., Nature 408, 86 (2000).

10. F. J. Slack et al., Mol. Cell 5, 659 (2000).

11. R. J. Johnston Jr., O. Hobert, Nature 426, 845 (2003).
12. S. Chang, R. J. Johnston Jr., C. Frøkjaer-Jensen, S. Lockery, O. Hobert, Nature 430, 785 (2004).

13. Y.-W. Hsieh, C. Chang, C.-F. Chuang, PLoS Genet. 8, e1002864 (2012).

14. Y. Zou, H. Chiu, D. Domenger, C.-F. Chuang, C. Chang, Sci. Signal. 5, ra43 (2012).

15. T. F. Duchaine et al., Cell 124, 343 (2006).

16. S. Y. Lin et al., Dev. Cell 4, 639 (2003).

17. A. E. Rougvie, V. Ambros, Development 121, 2491 (1995).

18. A. Rybak et al., Nat. Cell Biol. 11, 1411 (2009).

19. C. M. Hammell, I. Lubin, P. R. Boag, T. K. Blackwell, V. Ambros, Cell 136, 926 (2009).

Acknowledgments: We thank the $C$. elegans Genetic Center for $C$. elegans strains; the WormBase for readily accessible information; F. Ciamacco, B. Bayne, and C. Carrelli for technical assistance; A. Fire for C. elegans vectors; and V. Cleghon for critical reading of the manuscript. This work was funded by grants from the Whitehall Foundation (C.C. and C.-F.C.), the March of Dimes Foundation (C.C.), the Canada Foundation for Innovation (C.C.), by Alfred P. Sloan Research Fellowships to C.-F.C., by NIH grant R01 GM098026 to C.-F.C., and by NIH grant R01 GM034028-25 to V.A.

Supplementary Materials

www.sciencemag.org/cgi/content/full/340/6130/372/DC1 Materials and Methods

Figs. S1 to S12

Table S1

References (20-23)

10 October 2012; accepted 8 March 2013

$10.1126 /$ science. 1231321

\section{A Neural Marker of Perceptual Consciousness in Infants}

Sid Kouider, ${ }^{1,2 *}$ Carsten Stahlhut, ${ }^{2}$ Sofie V. Gelskov, ${ }^{1,3}$ Leonardo S. Barbosa, ${ }^{1}$ Michel Dutat, ${ }^{1}$ Vincent de Gardelle, ${ }^{1}$ Anne Christophe, ${ }^{1}$ Stanislas Dehaene, ${ }^{4,5,6,7}$ Ghislaine Dehaene-Lambertz ${ }^{5,6,7}$

Infants have a sophisticated behavioral and cognitive repertoire suggestive of a capacity for conscious reflection. Yet, demonstrating conscious access in infants remains challenging, mainly because they cannot report their thoughts. Here, to circumvent this problem, we studied whether an electrophysiological signature of consciousness found in adults, corresponding to a late nonlinear cortical response [ 300 milliseconds (ms)] to brief pictures, already exists in infants. We recorded event-related potentials while 5-, 12-, and 15-month-old infants $(N=80)$ viewed masked faces at various levels of visibility. In all age groups, we found a late slow wave showing a nonlinear profile at the expected perceptual thresholds. However, this late component shifted from a weak and delayed response in 5-month-olds (starting around $900 \mathrm{~ms}$ ) to a more sustained and faster response in older infants (around $750 \mathrm{~ms}$ ). These results reveal that the brain mechanisms underlying the threshold for conscious perception are already present in infancy but undergo a slow acceleration during development.

$\mathrm{R}$ ecent research shows that, in the first year of life, preverbal infants already display an impressive array of cognitive competences. For instance, their eye movements betray a capacity to monitor other people's beliefs at 7 months (1) and to draw probabilistic predictions about visual scenes at 12 months (2). Given these complex behaviors, one might consider it obvious that infants already have a conscious experience of their environment. However, this conclusion is unwarranted, because the presence of unconscious priming and "blind- sight" behaviors in normal and impaired adults $(3,4)$ shows that sophisticated processing can occur without consciousness.

How, then, might one test whether the brain mechanisms for conscious access are already present in infancy? Studying consciousness and its neural correlates in adults requires the collection of subjective reports of experience, classically through psychophysical paradigms contrasting visible and invisible stimuli (5). It is not impossible to obtain subjective reports from nonverbal organisms. For instance, monkeys can be trained to report the presence or absence of a stimulus, either by touching the location of a stimulus on a screen or by touching an alternative key to indicate that no stimulus had been presented. After a unilateral lesion in V1, they consistently press the "absent" key for stimuli contralateral to the lesioned side, although they remain able to localize them with high accuracy, which suggests that they undergo a "blindsight" phenomenon similar to that of human patients ( 6 ). However, it seems much more difficult to train infants to report similarly about their thoughts and percepts, which renders the issue of infant consciousness particularly challenging.

In this study, we follow an alternative strategy: examining whether the neural signatures of perceptual consciousness that are observed in adults can already be obtained in the developing brain. We capitalize on visual masking, a psychophysical phenomenon whereby a brief display, when followed by a second picture, vanishes from awareness. Previous research in adults has shown that the perception of masked displays

${ }^{1}$ Laboratoire de Sciences Cognitives et Psycholinguistique, EHESS/CNRS/ENS-DEC, 75005 Paris, France. ${ }^{2}$ Section for Cognitive Systems, Department of Informatics and Mathematical Modeling, Technical University of Denmark, 2800 Kongens Lyngby, Denmark. ${ }^{3}$ Danish Research Center for Magnetic Resonance, Copenhagen University Hospital, 2650 Hvidovre, Denmark. ${ }^{4}$ Collège de France, 75231 Paris, France. ${ }^{5}$ INSERM, U992, Cognitive Neuroimaging Unit, 91191 Gif-sur-Yvette, France. ${ }^{6} \mathrm{CEA}$, NeuroSpin Center, 91191 Gif-sur-Yvette, France. ${ }^{7}$ Université Paris Xl, 91405 Orsay, France.

*Corresponding author. E-mail: sid.kouider@ens.fr 
follows a two-stage process with distinct electrophysiological signatures $(7-10)$. During the first $\sim 200$ to $300 \mathrm{~ms}$ of processing, brain re- sponses increase linearly with the stimulus energy or duration. This early linear stage can be observed even on subliminal trials in which the
A

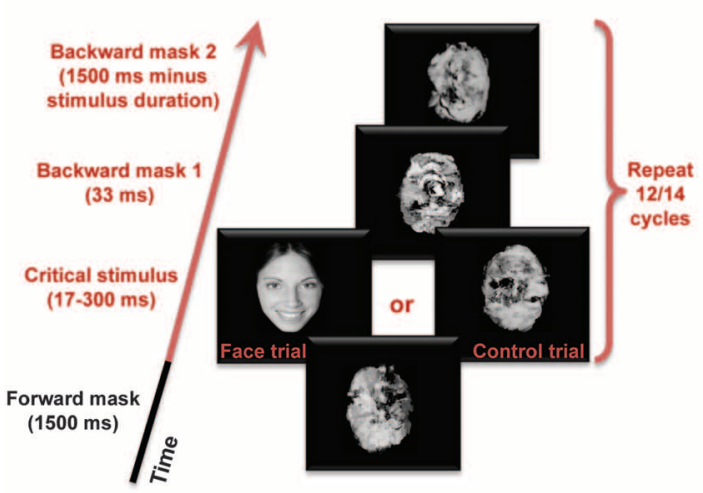

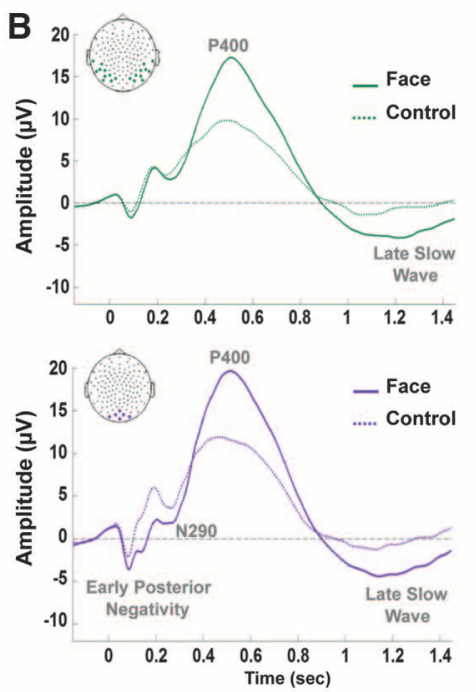

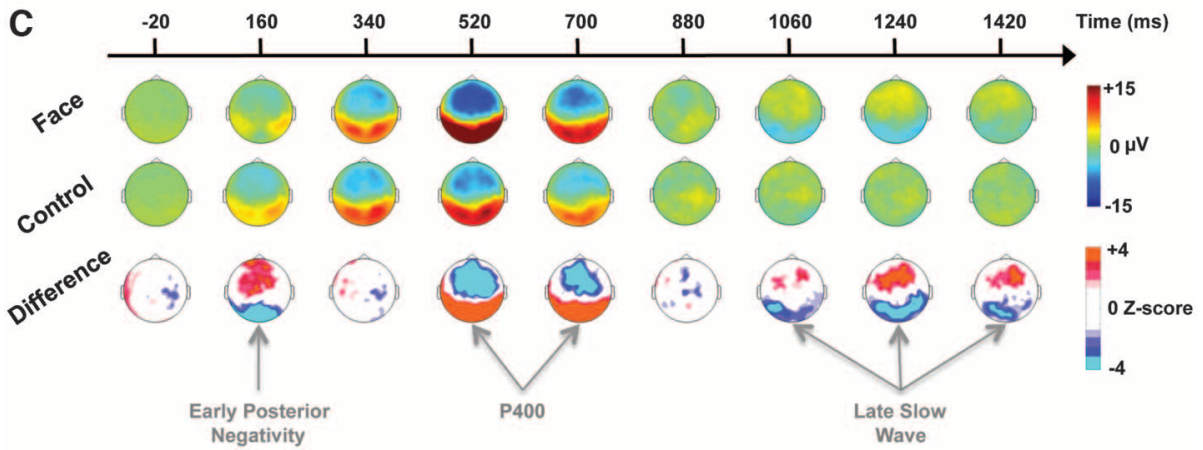

Fig. 1. (A) Schematic description of the procedure. Visibility was manipulated by presenting faces at various durations, within a series of masking patterns (scrambled layers of upside-down faces and objects) that prevented visual persistence and lingering afterimages. A control condition comprising exclusively scrambled patterns allowed for the localization of face-sensitive components. The younger (5-month-old) infants were tested with durations ranging from 50 to $300 \mathrm{~ms}$ in steps of $50 \mathrm{~ms}$. The older (12- and 15-month-old) groups were tested with face durations of 17, 33, 50, 100, 150, 200, and 250 ms. Infants received blocks with a forward mask followed by the random presentation of face and control trials at each duration, leading to 12 cycles in younger and 14 cycles in older infants. (B and C) Analysis of global ERPs (collapsing across the five durations common to all infants; i.e., from 50 to $250 \mathrm{~ms}$ ). (B) Grandaverage responses recorded over posterior medial occipital and lateral occipitotemporal electrodes and revealing the four components responding preferably to faces compared with scrambled controls (early posterior negativity, N290, P400, and LSW). (C) Two-dimensional (2D) scalp topographies over time showing the voltage ERP evoked by face and control trials, and their difference in terms of statistical significance maps (Z-scores). stimulus is subjectively invisible. By contrast, the second stage, which starts after $\sim 300 \mathrm{~ms}$, is characterized by a nonlinear, essentially all-ornone change in brain activity detectable with event-related potentials (ERPs) $(8,9,11)$ and intracranial recordings (12). Note that this second stage occurs specifically on trials reported as consciously seen [e.g., $(5,13,14)]$. During this stage, even a brief external stimulation, if it exceeds a certain threshold, can lead to a large and long-lasting pattern of activity involving the recruitment of a distributed parieto-frontal network, as well as the reactivation of the initial sensory regions. This sustained activity allows for the maintenance of the perceptual representation long after the stimulus is gone and coincides with subjective reports of visibility $(8,9)$.

Here, we examined whether the electrophysiological signatures of this two-stage process can be observed in infants. The presence of both early linear and late nonlinear electrophysiological markers during visual perception in infants would indicate that, first, the same architecture for perception is already at work at early stages of development and, second, that a distinction between nonconscious and conscious events may already be delineated in infants. We recorded high-density (128 electrodes) ERPs in three groups of infants [5-month-olds $(n=30), 12$-month-olds $(n=29)$, and 15-montholds $(n=21)]$ while they looked at masked faces presented at various durations, so as to induce different levels of visibility [see Fig. 1A and (15) for details].

Using the same stimuli in a behavioral study of preferential looking, we recently measured the masking threshold in infancy and evidenced important changes occurring at the end of the first year of life (16). Although 5- and 10-month-old infants oriented their gaze only to faces presented for $150 \mathrm{~ms}$ or longer, 15 -month-olds exhibited a perceptual threshold closer to the adult value (between 50 and $100 \mathrm{~ms}$ ). Furthermore, whereas 5- and 10-month-old infants oriented to suprathreshold faces in a purely reactive manner (i.e., performance systematically came back to the level of chance in between face occurrences, even though they were repeatedly presented at the same peripheral location on each trial), 15month-olds anticipated the face reappearance, which demonstrated that they were not only
Fig. 2. Event-related potentials as a function of age. Over time, 2D scalp topographies show statistical significance maps (Z-scores) of the face-control difference at 5, 12, and 15 months of age.

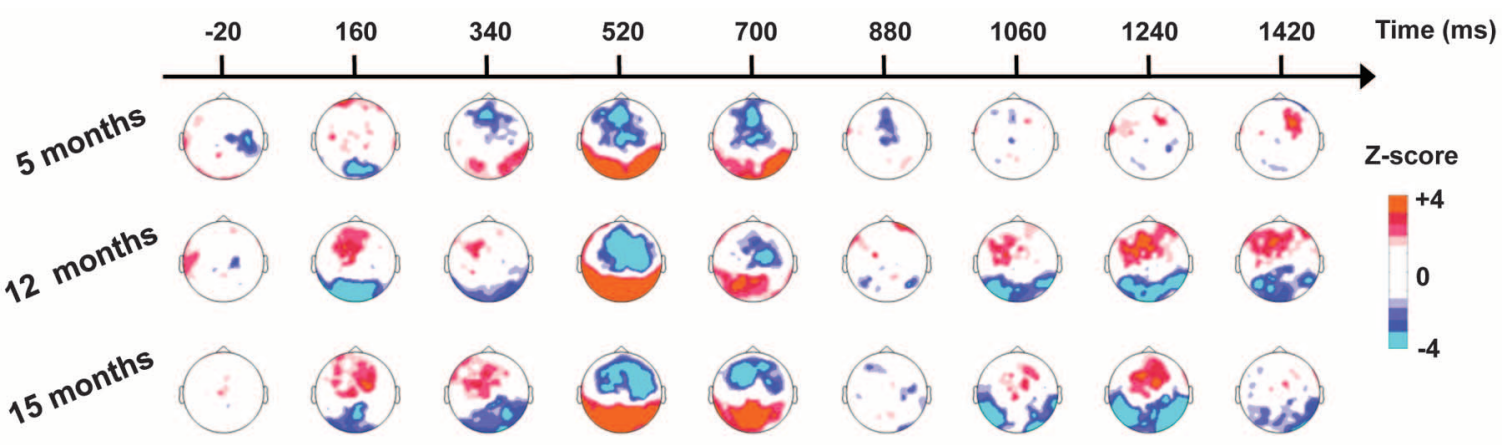


able to maintain the perceptual representation of a stimulus over time but also to use this information to adapt their behavior. At the brain level, we thus predicted a marked change around the end of the first year of life in infants. Specifically, we expected that the transition from linear brain responses to a sudden, nonlinear increase (a neural marker which in adults indicates the maintenance of perceptual information for conscious report) would be more pronounced and occur at shorter stimulus durations in the older babies.

We first extracted the event-related brain potentials evoked by masked faces relative to scrambled control stimuli. This first step allowed us to identify face-sensitive latencies and clusters of electrodes independently of infants' age and stimulus duration. Although faces were very briefly presented and embedded in masks, we observed the classical ERP components of face perception in infants, with a series of four components responding preferably to faces (Fig. 1, $\mathrm{B}$ and $\mathrm{C}$ ). The first difference was an early posterior negativity (EPN) peaking around $150 \mathrm{~ms}$ $[t(79)=6.38, P<0.001]$ over medial occipital electrodes. This component is likely to reflect the earliest bottom-up stage, where structured images are separated from scrambled stimuli (17). It was followed by two components classically associated with face processing in infants $(18,19)$ : an $\mathrm{N} 290$ over the same posterior occipital electrodes $[t(79)=2.35, P<0.05]$ and then a P400 extending bilaterally from occipital to temporal regions $[t(79)=11.29, P<0.001]$. Although the homology of these components with the N170 specifically elicited by faces in adults remains debated, the N290 is sensitive to the presence of contrasted human eyes, whereas the P400 depends on infants' knowledge of the prototypical face configuration $(18,19)$. Finally, we observed a sustained late negativity starting from $900 \mathrm{~ms}$ over the same occipito-temporal electrodes, along with a positivity over anterior electrodes $[t(79)=4.75, P<0.001]$. This late response corresponds to the late slow wave (LSW), which has been linked to stimulus encoding and recognition memory but also more generally to attention and novelty detection (18-21).

We then examined how these components evolved with age (Fig. 2 and figs. S1 to S3). The EPN and P400 components were significant in all age groups (all $P$ values were $<0.01$ ) and were of equivalent magnitude across the three age groups (both $F$ values were $<1$ ) (see fig. S1). By contrast, we found robust interactions with age for the N290 $\left(F_{2,77}=10.17, P<0.001\right)$ and for the $\operatorname{LSW}\left(F_{2,77}=4.17, P=0.019\right)$, which suggested the presence of a developmental change for these two components. Indeed, for both components, the difference between faces and controls occurred solely in 12-month-olds (both $P$ values were $<0.001$ ) and 15 -month-olds (both $P$ values were $<0.005$ ), with the same amplitude for both groups $(F<1)$.

Having established which components were sensitive to faces, we studied their variation as a function of stimulus duration in the face trials. Because the results for the groups of 12- and 15-month-olds were virtually identical, they were collapsed to increase signal-to-noise ratio. The waveforms elicited by faces at each stimulus duration are shown in Fig. 3 (see fig. S4 for control stimuli and difference waves). In the older group of 12- to 15-month-olds, it is apparent that the N290, P400, and LSW components are strongly and monotonically modulated by stimulus duration. Furthermore, the LSW is essentially absent for face presentations of up to $50 \mathrm{~ms}$ duration and suddenly jumps to a strong negativity for durations of $100 \mathrm{~ms}$ and beyond. This is consistent with our previous behavioral study, which suggests a perceptual threshold around 50 to $100 \mathrm{~ms}$ of presentation duration (16). To evaluate these points statistically, we submitted each electrode and time point of the face conditions to two contrasts (8). The first was a linear contrast eval- uating a continuous variation in voltage with stimulus duration. The second was nonlinear and tested for a jump around the presumed threshold, i.e., a larger increase in voltage when presentation duration moved from 50 to $100 \mathrm{~ms}$, compared with the other ranges of stimulus duration (average change in voltage from 16 to $50 \mathrm{~ms}$ and from 100 to $250 \mathrm{~ms}$ ). The results confirmed the presence of both linear and nonlinear effects over the same occipito-temporal electrodes, but with distinct time courses (Fig. 4). Although the linear effect became significant relatively early at $180 \mathrm{~ms}$, the nonlinear trend emerged rather abruptly only at a later stage, around $750 \mathrm{~ms}$ (Fig. 4, C and D). Furthermore, whereas the N290 and $\mathrm{P} 400$ increased in a strictly linear manner, both linear and nonlinear contrasts were significant for the LSW. This joint significance was due to a sigmoidal response profile: The LSW showed a general increase with stimulus duration, but with a large nonlinear jump around the previously identified perceptual threshold (from 50 to $100 \mathrm{~ms}$ ). These results, which dissociate early linear and late nonlinear components, demonstrate that an adultlike, although much slower, sequence of perceptual stages is already observed by the end of the first year of life.

What about younger infants? In comparison with the older group, modulations of voltage by face duration were overall smaller in 5-montholds (Fig. 3A). Nevertheless, the linear contrast again achieved significance for the N290, the P400, and even for the LSW (Fig. 4, A to C). Although no significant LSW difference wave was found at this age in the global analysis (i.e., collapsing stimulus durations) (see Fig. 2 and text above), an LSW component was actually present and differed significantly from baseline at face durations of $150 \mathrm{~ms}$ and above (all $P$ values were $<0.002$ ) (Fig. 3A). Again, this is consistent with our previous behavioral study showing that 5-month-old infants did not orient to faces presented for duration of $100 \mathrm{~ms}$ or less
Fig. 3. (A and B) Variation of ERPs with stimulus duration in younger and older infants. Graphs show the grand-average responses recorded over occipitotemporal electrodes for faces as a function of stimulus duration.
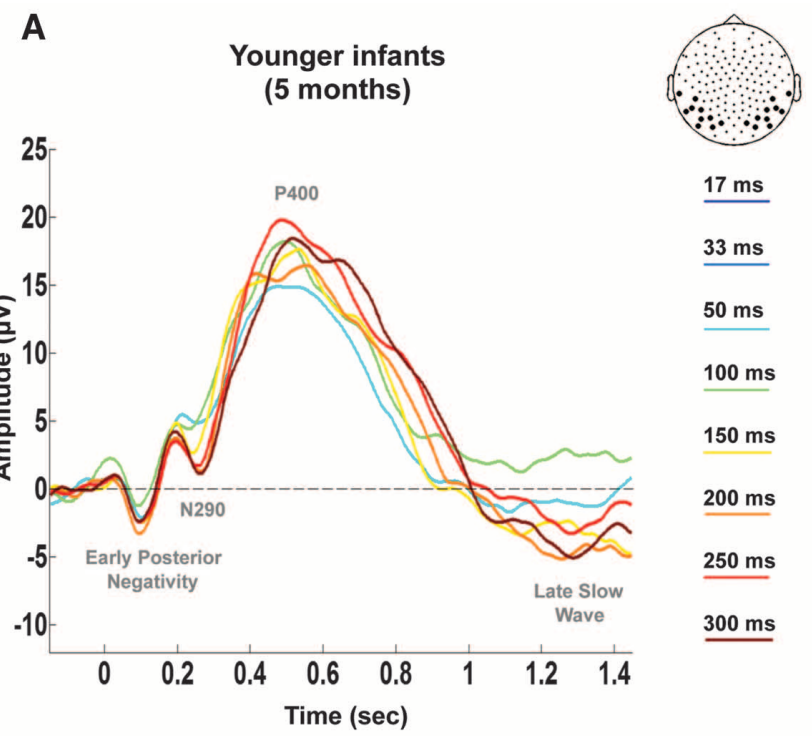

B
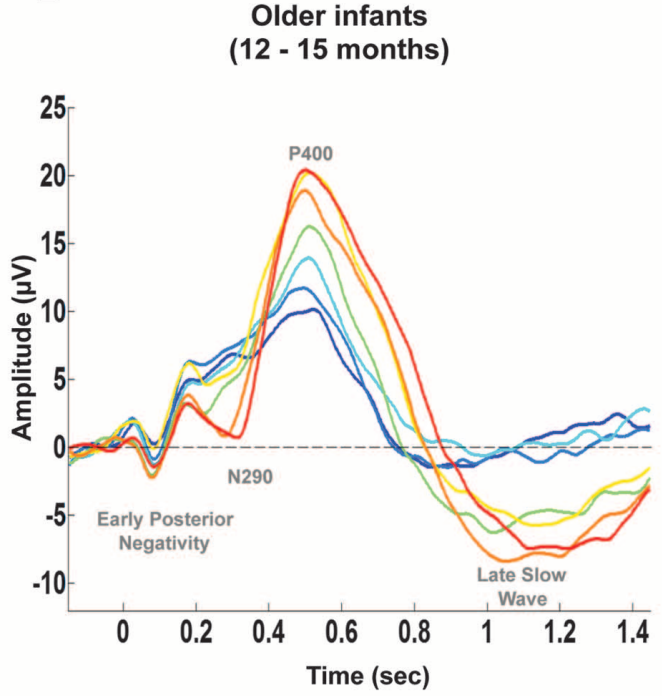
but showed an abrupt increase in responding to faces from 100 to $150 \mathrm{~ms}$ (16). Indeed, our contrast testing for a nonlinear response component occurring between the predicted durations of $100 \mathrm{~ms}$ and $150 \mathrm{~ms}$ began to deviate from baseline abruptly around $900 \mathrm{~ms}$, simultaneously with the LSW (Fig. 4, B to D). However, we observed that the LSW component was slow and small at this age, and statistical significance of the nonlinearity only attained in the latest period of the epoch (starting at $1320 \mathrm{~ms}$ ). Thus, we found that the two-stage architecture for perception can be evidenced early on during development, in infants as young as 5 months, although this distinction was not as clearly delineated as for older infants.

Because the LSW exhibits a late nonlinearity that matches the visibility thresholds established in previous behavioral testing (10), we suggest that this electrophysiological component constitutes a reliable neural index of conscious access to perceptual information in preverbal infants. Note that this late component cannot be ascribed to unmatched number of trials or larger movement artifacts in one specific condition [see (15) for details]. LSWs, generally recorded after $600 \mathrm{~ms}$, have been first related to memory encoding and recognition $(18,19)$. Since then, slow waves have been described in different visual and auditory paradigms in awake and attentive infants $(20,21)$. For instance, the LSW is observed after a deviant sound in awake, but not asleep, infants (22). It also reflects the infant's allocation of attention toward the mother's face, a familiar toy, or a novel event against a background of familiar or partially familiar events $(18,20)$. The diversity of experimental paradigms eliciting LSWs in infants suggests that this component reflects a generic amodal system involving higher-order processes, such as attention and working memory. Because of these functional characteristics, equivalence with the P300 in adults has been proposed (23). Our results support this interpretation by showing that the LSW exhibits the same nonlinear response profile as the P300 and follows earlier components responding in a linear fashion as a function of stimulus intensity $(8,11)$. Note that the adult P300 component strongly correlates with subjective reports of visibility $(5,8,9)$ and is therefore thought to constitute a neural signature of access to consciousness. The functional similarity of infant and adult responses, combined with our previous behavioral results (16), suggests that a stage of conscious processing already exists in infants and that the LSW constitutes a reliable neural signature of consciousness in infants.
Nonetheless, our results also reveal that this neural marker of consciousness in infants is triggered at a much later time than in adults. Indeed, whereas the nonlinear response to masked stimuli is found around $300 \mathrm{~ms}$ in the adult brain $(8,11)$, here, we observed it around $750 \mathrm{~ms}$ in 12- to 15-month-old infants, and after $900 \mathrm{~ms}$ or more in 5-month-olds. Hence, this second processing stage is disproportionately slow in infants and remains so for many months.

What constitutes the neural basis of this exceedingly slow response? The first stages of perception (indexed by the EPN and P400 components), which exhibited equivalent latencies in all age groups, probably reflect the rapid maturation of the sensory visual system during the first few months of life (24). By contrast, the long latencies of the LSW are compatible with the notion that this nonlinear response depends on a large-scale distributed network of long-distance corticocortical connections (5). Most of these long-fiber tracts are present in infants (25) and even fetuses (26), but they remain weakly myelinated through the first year of life (24). Such a weak myelination could explain why the LSW emerged later in 5-montholds compared with older infants. It would lead to a reduced and slower interplay between sensory regions and associative parietal-prefrontal

\section{Linear increase}
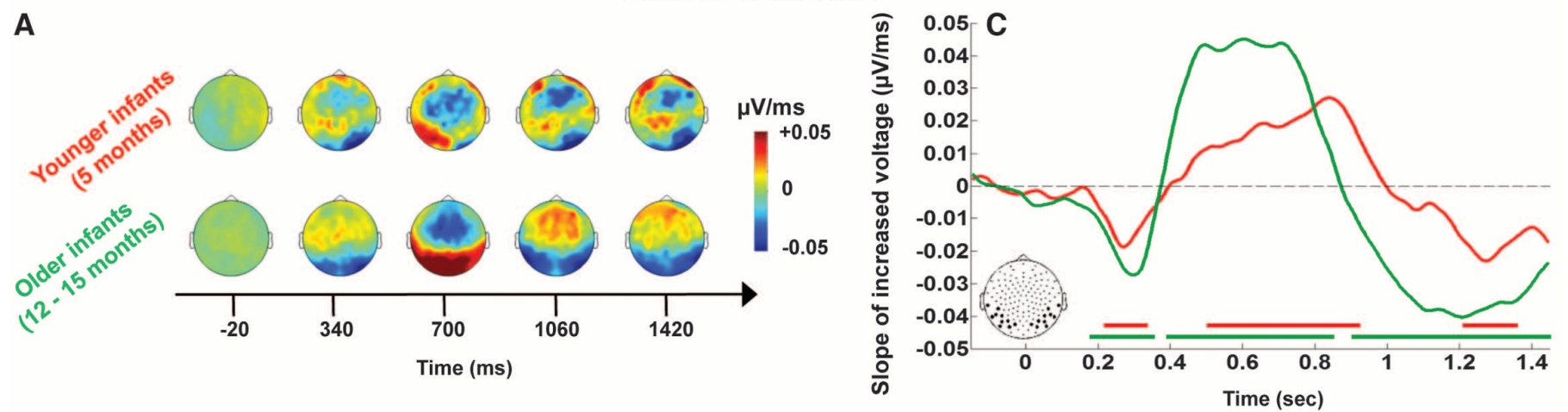

Non-linear increase
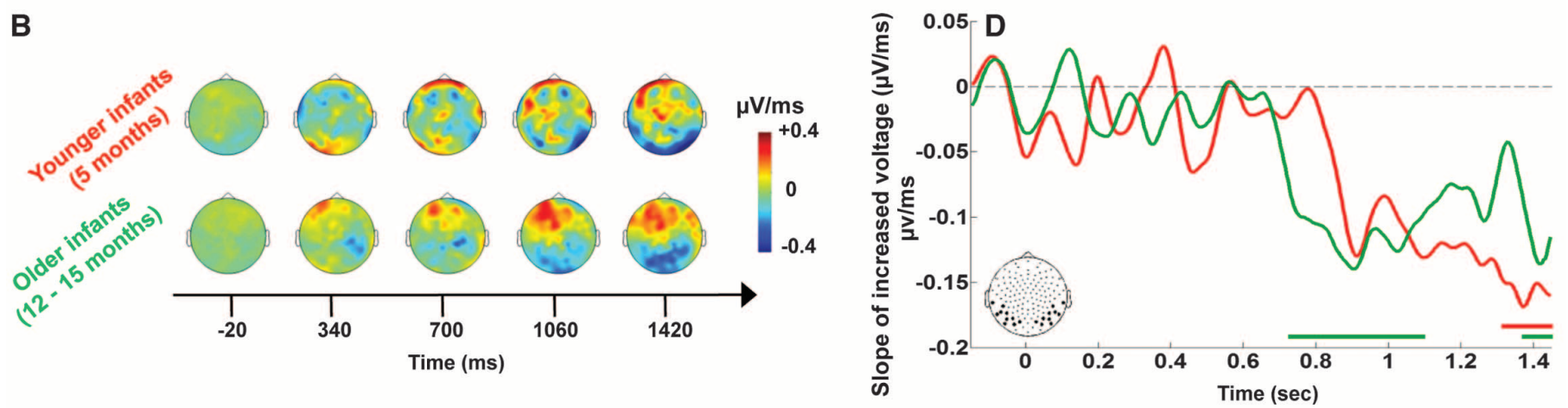

Fig. 4. (A to D) Time course of the linear and nonlinear increases in brain activity as a function of face duration, in younger and older infants. The 2D plots show the scalp topographies of the linear and nonlinear contrasts. The unit of measurement is the slope with which voltage increases

with stimulus duration, expressed in units of microvolts per millisecond. Response profiles over occipitotemporal electrodes are accompanied with horizontal bars indicating temporal clusters of 10 consecutive significant time points (40 ms). 
areas, which limits the functional connectivity that is necessary for the maintenance and flexible use of sensory information. Consistent with this interpretation, the LSW scalp topography, showing posterior negativities over bilateral occipitotemporal regions, is suggestive of a late reactivation of sensory regions. In adults, such a late reactivation has been argued to reflect topdown mechanisms of attentional amplification that allow for the maintenance of a stimulus representation over time that is characteristic of perceptual consciousness $(5,7,9)$. Another potential factor is the protracted dendritic development and synaptogenesis in associative areas (27). Indeed, the prefrontal cortex shows a marked maturation and massive reorganization at the end of the first year of life, resulting in dramatic improvements in a wide range of cognitive abilities at that age (28). In any case, the acceleration of information transfer between associative and sensory areas might be a key factor underlying infants' improved perceptual efficiency across development.

In sum, our data indicate that infant perception is organized into a series of stages similarly to adult perception; these include, crucially, a late nonlinear stage that, in adults, systematically accompanies reports of conscious perception and, in infants, correlates with psychophysical thresholds for orienting to masked stimuli. We propose that this late nonlinear response constitutes a new, specific, and objectively measurable candidate marker that putatively reflects conscious perception. It is important to acknowledge that our research does not provide a direct proof of subjective experience. Indeed, it is a genuine philosophical problem whether such a proof can ever be obtained from purely objec- tive neurophysiological data. Rather, we show that neural markers of consciousness found in adults can be generalized to infant populations. Such objective measures have proven useful to probe consciousness in patients in a vegetative state and in minimally conscious patients (29) and might help pediatricians confront issues of infant consciousness in relation to anesthesia, pain, and pathologies.

\section{References and Notes}

1. A. M. Kovács, E. Téglás, A. D. Endress, Science 330 , 1830 (2010).

2. E. Téglás et al., Science 332, 1054 (2011).

3. S. Kouider, S. Dehaene, Philos. Trans. R. Soc. London B Biol. Sci. 362, 857 (2007).

4. L. Weiskrantz, Consciousness Lost and Found: A Neuropsychological Exploration (Oxford Univ. Press, New York, 1997).

5. S. Dehaene, J. P. Changeux, Neuron 70, 200 (2011).

6. A. Cowey, P. Stoerig, Nature 373, 247 (1995).

7. V. A. Lamme, P. R. Roelfsema, Trends Neurosci. 23, 571 (2000).

8. A. Del Cul, S. Baillet, S. Dehaene, PLoS Biol. 5, e260 (2007).

9. C. Sergent, S. Baillet, S. Dehaene, Nat. Neurosci. 8, 1391 (2005).

10. M. M. Chun, M. C. Potter, J. Exp. Psychol. Hum. Percept. Perform. 21, 109 (1995).

11. L. Melloni et al., J. Neurosci. 27, 2858 (2007).

12. R. Q. Quiroga, R. Mukamel, E. A. Isham, R. Malach, I. Fried, Proc. Natl. Acad. Sci. U.S.A. 105, 3599 (2008).

13. E. K. Vogel, S. J. Luck, K. L. Shapiro, J. Exp. Psychol. Hum. Percept. Perform. 24, 1656 (1998).

14. S. Kouider, S. Dehaene, in Mental Processes in the Human Brain, J. Driver, P. Haggard, T. Shallice, Eds. (Oxford Univ. Press, Oxford, 2007), pp. 155-185.

15. Materials and methods are available as supplementary materials on Science Online.

16. S. V. Gelskov, S. Kouider, Cognition 114, 285 (2010).

17. B. Rossion, S. Caharel, Vision Res. 51, 1297 (2011)

18. M. de Haan, C. A. Nelson, Dev. Psychol. 35, 1113 (1999).
19. M. de Haan, M. H. Johnson, H. Halit, Int. J. Psychophysiol. 51, 45 (2003).

20. G. D. Reynolds, J. E. Richards, Dev. Psychol. 41, 598 (2005)

21. G. Csibra, E. Kushnerenko, T. Grossmann, in Handbook of Developmental Cognitive Neuroscience, C. A. Nelson and M. Luciana, Eds. (MIT Press, Cambridge, MA, ed. 2, 2008), pp. 247-262.

22. A. D. Friederici, M. Friedrich, C. Weber, Neuroreport 13 1251 (2002)

23. C. A. Nelson, K. M. Thomas, M. de Haan, S. S. Wewerka, Int. J. Psychophysiol. 29, 145 (1998).

24. P. Yakovlev, A. R. Lecours, in Regional Development of the Brain in Early Life, A. Minkovski, Ed. (Blackwell, Oxford, 1967), pp. 3-69.

25. ]. Dubois, L. Hertz-Pannier, G. Dehaene-Lambertz, Y. Cointepas, D. Le Bihan, Neuroimage 30, 1121 (2006).

26. E. Takahashi, R. D. Folkerth, A. M. Galaburda, P. E. Grant, Cereb. Cortex 22, 455 (2012).

27. M. J. Koenderink, H. B. Uylings, Brain Res. 678, 233 (1995).

28. A. Diamond, P. S. Goldman-Rakic, Exp. Brain Res. 74, 24 (1989).

29. T. A. Bekinschtein et al., Proc. Natl. Acad. Sci. U.S.A. 106, 1672 (2009).

Acknowledgments: This research was supported by funding from the Agence National de la Recherche and from the European Research Council ("DynaMind" project) to S.K., from the Lundbeck Foundation (CIMBI project) to C.S. and from the McDonnell foundation to G.D.-L. We thank C. Summerfield for suggestions on the manuscript; A.-C. Fievet, and I. Vendelin for help with the data collection; and C. Billard (CHU Kremlin-Bicêtre), D. Chabrol (Hôpital Cochin), and L. K. Hansen (Danish Technical University) for their support to our research.

\section{Supplementary Materials}

www.sciencemag.org/cgi/content/full/340/6130/376/DC1

Materials and Methods

Supplementary Tex

Figs. S1 to $\mathrm{S} 5$

References (30-32)

8 November 2012; accepted 19 February 2013 10.1126/science.1232509 